\title{
Tellus
}

\section{Sea surface temperature anomalies in the North Pacific Ocean}

\section{Richard W. Reynolds}

To cite this article: Richard W. Reynolds (1978) Sea surface temperature anomalies in the North Pacific Ocean, Tellus, 30:2, 97-103, DOI: 10.3402/tellusa.v30i2.10321

To link to this article: https://doi.org/10.3402/tellusa.v30i2.10321
(c) 1978 The Author(s). Published by Taylor and Francis Group LLC

曲 Published online: 15 Dec 2016.

Submit your article to this journal $\pi$

山 Article views: 67

Q View related articles $₫$

4 Citing articles: 3 View citing articles 둔 


\title{
Sea surface temperature anomalies in the North Pacific Ocean
}

\author{
By RICHARD W. REYNOLDS, ${ }^{1}$ Max-Planck-Institut für Meteorologie, 2 Hamburg 13, FRG
}

(Manuscript received August 1; in final form November 18, 1977)

\begin{abstract}
Sea surface temperature anomalies in the mid-latitude North Pacific Ocean were compared with a first-order autoregression model in which the anomalies are forced by local atmospheric white noise. The results showed that the model can explain the power spectrum of the anomalies for a little over $50 \%$ of the investigated regions, mainly in the central regions of the Pacific, but fails, not surprisingly, in regions of strong oceanographic processes.
\end{abstract}

\section{Introduction}

Hasselmann (1976) has proposed a stochastic model of climate variability in which slow changes of climate are explained as the integral response of the climate to continuous random excitation by shorter time-scale disturbances. The entire climate system is divided into two subsystems with widely differing time scales: a rapidly varying "weather" subsystem and a slowly responding "climate" subsystem. The "weather" components are treated as random (white noise) forcing terms. The "climate" components then act as integrators of this excitation. The model predicts that the climate components should exhibit a red power spectrum which for intermediate frequencies is inversely proportional to the frequency squared.

Frankignoul and Hasselmann (1977) have applied the model to a simplified atmospheric and oceanic system. In this system the climatic components were represented as sea surface temperature (SST) anomalies which were driven by uncorrelated white-noise atmospheric forcing. To balance the atmospheric input and maintain a statistically stationary response, a negative stabilizing feedback, characterized by a linear feedback factor, $\lambda$, was added where $(2 \pi \lambda)^{-1}$ is the $e$-folding

\footnotetext{
1 Present address: Climatic Research Institute, Oregon State University, Corvallis, Oregon, 97331.
}

decay time. With feedback, the SST anomalies have a spectral form, $\hat{E}(f)$, given by:

$E(f)=\frac{A}{f^{2}+\lambda^{2}}$,

where $f$ is frequency and $A$ is proportional to the variance of the white-noise input spectrum. Equation (1) can be recognized as the spectrum of a first-order autoregression process with random noise.

Frankignoul and Hasselmann (1977) verified (1) by numerical experiments and by comparison with individual weather ship and integrated North Pacific data. In this paper a more detailed regional study of the North Pacific Ocean is presented. Equation (1) is compared with measured SST anomalies for five degree squares to determine in which regions the stochastic climate model is applicable.

\section{Procedure}

For comparison with real data, (1) must be expressed in terms of the spectrum that would be determined for a discrete time series of sampling interval, $\Delta t$. Following Wunsch (1972), (1) becomes:

$\hat{E}_{1}(f)=\sum_{m=-\infty}^{\infty} \frac{A}{\left(f-2 m f_{n}\right)^{2}+\lambda^{2}}$, 
where $f_{n}=1 /(2 \Delta t)$ is the Nyquist frequency, $m$ is an integer and $0 \leq f \leq f_{n}$. The total variance, $\sigma$, of (1) and (2) is conserved since:

$\sigma=\int_{0}^{\infty} \hat{E}(f) d f=\int_{0}^{f_{n}} \hat{E}_{1}(f) d f=\frac{A \pi}{2 \lambda}$.

With the aid of Cauchy's integral theorem of residues (cf. Copson, 1960, p. 144), (2) can be expressed more simply as:

$E_{1}(f)=\frac{N_{1}}{1+a_{1}^{2}-2 a_{1} \cos \left(\pi f / f_{n}\right)}$,

where

$\alpha_{1}=\exp \left(-\pi \lambda / f_{n}\right)$,

and

$N_{1}=A \frac{\pi \alpha \sinh \left(\pi \lambda / f_{n}\right)}{\lambda f_{n}}$.

Equation (3) is the spectrum of a discrete firstorder autoregression process which is forced by white noise (cf. Jenkins and Watts, 1968) where $\alpha_{1}$ is the autocorrelation of the first lag and $N_{1}$ is the variance of the noise.

The measured SST anomalies were obtained from NORPAX data of the mid-latitude $\left(20^{\circ} \mathrm{N}-\right.$ $55^{\circ} \mathrm{N}$ ) North Pacific. The data consisted of $28 \frac{1}{2}$ years of monthly average temperatures for a fivedegree square grid. To deal with occasional data gaps, each time series was divided into as many subseries of 64 consecutive points as possible. If at least three subseries were obtained for each grid point, the Fourier coefficients for each subseries were computed by a fast Fourier transform. A discrete spectrum, $E\left(f_{j}\right)$, was then calculated from the square of the average of the Fourier coefficients at each frequency. Since the resulting number of spectral degrees of freedom can be shown (Hasselmann, personal communication) to be two times the number of subseries used, $E\left(f_{j}\right)$ had six to maximally ten degrees of freedom. The spectral resolution was 0.19 cycles per year (cpy); the Nyquist frequency was 6 cpy.

To verify the stochastic model for SST anomalies, it must be shown that (4) is an adequate representation of $E\left(f_{j}\right)$ within the statistically prescribed error limits. This was done by determining the two free parameters in (4), $\alpha_{1}$ and $N_{1}$, at each grid location, by minimizing the deviation, $\varepsilon$, between $E$ and $E$ by a least square fit:

$\varepsilon=\sum_{j=0}^{p-1}\left[\ln E\left(f_{j}\right)-\ln E\left(f_{j}\right)\right]^{2}=\min$,

where the number of spectral points, $p=33$. A logarithm of the spectrums was used in the definition of $\varepsilon$ so that the statistical errors for each spectral frequency would receive equal weight in (7).

A similar least square fit was also made for two extensions of (4), each of which contained three free parameters. The first, $E_{2}(f)$, included an extra noise term, $M$, such that:

$\hat{E}_{2}(f)=\frac{N_{2}}{1+\alpha_{2}^{2}-2 a_{2} \cos \left(\pi f / f_{n}\right)}+M$.

The second, $\hat{E}_{3}(f)$, represented the spectrum of a second-order autoregression process (Jenkins and Watts, 1968):

$\hat{E}_{3}(f)=$

$\frac{N_{3}}{1+\alpha_{3}^{2}+\beta^{2}-2 \alpha_{3}(1-\beta) \cos \left(\pi f / f_{n}\right)-2 \beta \cos \left(2 \pi f / f_{n}\right)}$,

where $\beta$ is the second-order regression coefficient. In all cases the free parameters $\left(\alpha_{1}, N_{1}\right)$; $\left(\alpha_{2}, N_{2}, M\right)$; and $\left(\alpha_{3}, N_{3}, \beta\right)$ were determined independently of each other by minimizing $\varepsilon$ in (7) for each model spectrum.

To determine which of the model spectrums were statistically significant, the hypothesis was made that the model fits the true spectrum perfectly. However, because of the statistical finite-sampling error, the model spectrum will never fit the measured spectrum exactly, and consequently $\varepsilon$ is never zero. The probability distribution of $\varepsilon$ is a $\chi^{2}$ distribution with $p-q$ degrees of freedom where $q$ is the number of fitted parameters in the model spectrum. Since $p-q$ is large the distribution can be approximated as a Gaussian with a mean and variance (see Appendix) given by:

$$
\begin{aligned}
& \langle\varepsilon\rangle=\frac{2}{v}(p-q), \\
& \sigma_{\varepsilon}^{2}=\left\langle(\varepsilon-\langle\varepsilon\rangle)^{2}\right\rangle=\frac{8}{v^{2}}(p-q),
\end{aligned}
$$

where $v$ is the number of spectral degrees of freedom of $E\left(f_{j}\right)$. A critical $\varepsilon$, $\bar{\varepsilon}$, was then defined 
such that the probability that $\varepsilon<\bar{\varepsilon}$ is $95 \%$ :

$\bar{\varepsilon}=1.65 \sigma_{\varepsilon}+\langle\varepsilon\rangle$.

For each fitted spectrum the model was considered statistically significant, within a $95 \%$ confidence limit, if $\varepsilon<\bar{\varepsilon}$.

\section{Results}

The boxed-in region in Fig. 1 indicates the fivedegree squares for which measured spectrums were computed. For each position, $\varepsilon$ was determined for

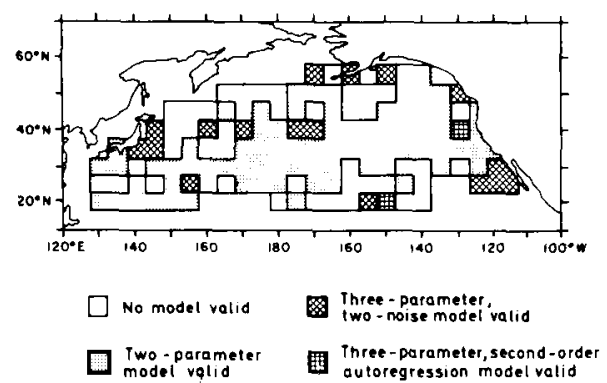

Fig. 1. The regions of validity of the three spectral models (see text).

the two-parameter model (4). The regions in which the fit was significant $(\varepsilon<\bar{\varepsilon})$ are dotted. In the remaining regions, the $\varepsilon$ 's for both three-parameter models were computed. If both models were significant, the model with the smaller $\varepsilon$ was chosen as the "valid" model. An example of the three fitted model spectrums and the measured spectrum is shown in Fig. 2. Here only the two three-parameter models were significant.

The regions in which the two-parameter model is not valid (statistically significant) can generally be related to known areas of strong oceanographic processes, such as the Arctic and subtropic convergences. In these regions oceanographic processes are apparently at least as important as the local atmospheric forcing as the cause of SST variability. However, in the remaining "quiet" areas of the ocean $(54 \%$ of the total), the two-parameter stochastic-forcing model is validated.

The three-parameter models are jointly valid over 20 of the remaining five-degree squares (14\% of the total). Although the regions are scattered, they occur principally along the course of the Kuroshio and off the coast of North America. The

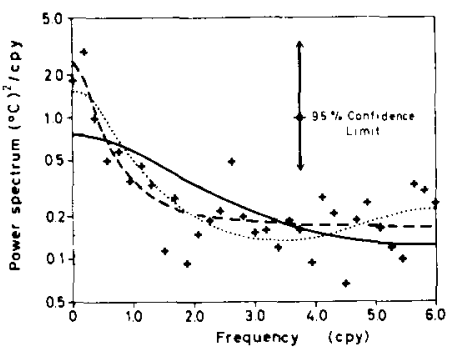

Fig. 2. A sample SST anomaly power spectrum with eight degrees of freedom at $40^{\circ} \mathrm{N}$ latitude and $145^{\circ} \mathrm{E}$ longitude. A plus means the measured spectrum, the full line two-parameter model, the dashed line the threeparameter, two-noise model and the dotted line the three-parameter, second-order autoregression model.

three-parameter, two-noise model was generally better (smaller $\varepsilon$ ) than the second-order autoregression model. As can be seen in Fig. 1, the second-order autoregression model was found to be better for only two squares (1.4\% of the total).

For the valid two-parameter fits of (4), the value of $\lambda$ was calculated from (5). The mean value, $\bar{\lambda}$, was found to be $1.1 \mathrm{yr}^{-1}$. In Fig. 3, the regions are shown where $\lambda$ differed from $\lambda$ by more than $15 \%$ and more than $30 \%$. It can be seen that the regions of large and small $\lambda$ can be roughly divided by the $165^{\circ} \mathrm{W}$ meridian. This may indicate that the Northern Pacific SST anomalies have more thermal inertia in the eastern part.

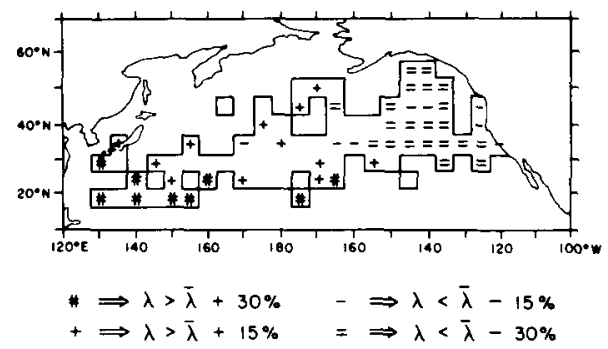

Fig. 3. Regions where the two-parameter model's $\lambda$ differs from $\bar{\lambda}\left(=1.1 \mathrm{yr}^{-1}\right)$. The rectangular lines indicate the boundaries of the valid two-parameter fit from Fig. 1.

The value $\bar{\lambda}=1.1 \mathrm{yr}^{-1}$ is larger than the value $0.32 \mathrm{yr}^{-1}$ determined by Frankignoul and Hasselmann (1977) for the first empirical orthogonal function (EOF) of Davis (1976) for the same data set. The difference may be attributed to the EOF 
procedure, which tends to assign the features of shorter wavelengths to higher-order functions. This eliminates much of the short-period noise from the lower orthogonal functions and characteristically lowers the value of $\lambda$. To illustrate this effect, the two-parameter model was fitted to the spectrum of the first fifteen EOF. The $\lambda$ 's for statistically valid fits are shown in Fig. 4. As expected the value of $\lambda$ has increased with increasing EOF order until $\lambda$ for the tenth EOF is within $10 \%$ of $\bar{\lambda}$.

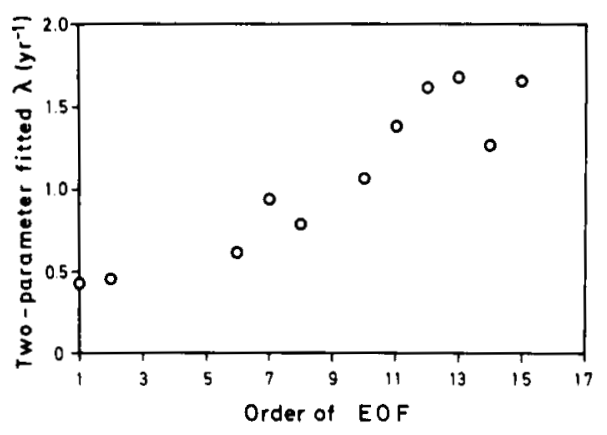

Fig. 4. Values of $\lambda$ for a statistically valid fit for the empirical orthogonal functions (EOF).

For the valid two-parameter fits, the value of $A$ was determined from (6). In Fig. 5 the regions are indicated in which $A$ differed from the mean, $\bar{A}$, by more than $15 \%$ and $30 \%$. Since $A$ is proportional to the white-noise forcing, two regions of strong forcing are indicated. The more important region is at $40^{\circ} \mathrm{N}$ between approximately $170^{\circ} \mathrm{E}$ and $160^{\circ} \mathrm{W}$.

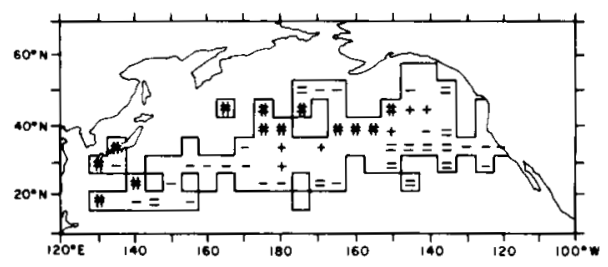

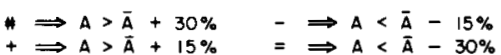

Fig. 5. Regions where the two-parameter model's $A$ differs from $A\left(=0.30\left({ }^{\circ} \mathrm{C}\right)^{2} \mathrm{cpy}\right)$. See also Fig. 3 .

The variance, $\sigma$, of the SST anomaly signal which can be attributed to the valid two-parameter fit can now be computed by (3). The distribution of $\sigma$ is shown in Fig. 6 for regions in which $\sigma$ differs from the mean value, $\bar{\sigma}$, by more than $15 \%$ and

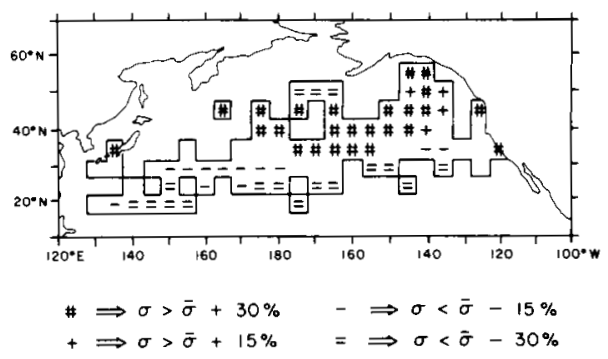

Fig. 6. Regions where the two-parameter model's variance, $\sigma$, differs from $\bar{\sigma}=\left(0.45\left({ }^{\circ} \mathrm{C}\right)^{2}\right)$. See also Fig. 3 .

$30 \%$. For comparison, Fig. 7 shows the distribution of the total SST anomaly variance for the entire region studied. It can be seen that regions of large $\sigma$ and large total variance are very similar.

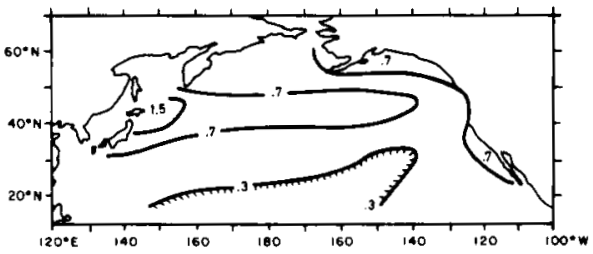

Fig. 7. Distribution of the variance of SST anomalies in $\left({ }^{\circ} \mathrm{C}\right)^{2}$. The mean of the variance is $0.58\left({ }^{\circ} \mathrm{C}\right)^{2}$.

The boundaries of the region of validity of the two-parameter model can also be determined from the sensitivity of the estimates of the model parameters to the least squares fit procedure. For example, an alternate estimate of $\alpha$ can be obtained by least square fitting a first-order autoregression process directly to the original time series of SST anomalies. This yields an $a$ value equal to the first lagged auto-correlation (Jenkins and Watts, 1968); the feedback parameter can then be determined from (5). A relative error, $\gamma$, can now be defined as:

$\gamma=\left|\frac{\lambda-\lambda_{\mathrm{ac}}}{\lambda}\right|$

where $\lambda$ is the feedback parameter from the twoparameter model and $\lambda_{\mathrm{ac}}$ is the feedback parameter determined from the auto-correlation. Values of $\gamma$ were determined for all points irrespective of whether $\varepsilon<\bar{\varepsilon}$ and are shown in Fig. 8. Large values of $\gamma$ are seen to coincide with the previously defined boundaries limiting the region of validity of the local stochastic forcing model. 


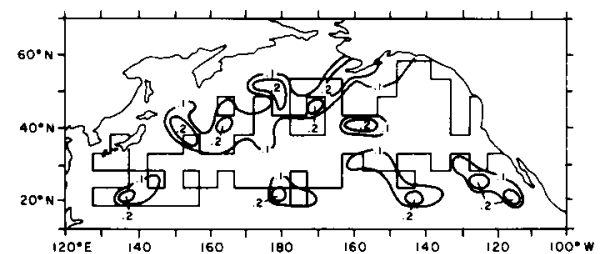

Fig. 8. Distribution of the relative error between the spectral two-parameter fitted $\lambda$ and the fitted $\lambda_{\mathrm{ac}}$ from the first-lagged autocorrelation. See also Fig. 3.

\section{Summary}

A little more than $50 \%$ of the mid-latitude North Pacific SST anomalies can be represented as a firstorder autoregression process (the two-parameter model), i.e. as the response of a system with negative linear feedback to white atmospheric forcing. The average feedback parameter, $\lambda$, was found to be about $1 \mathrm{yr}^{-1}$, or equivalently, the SST anomaly $e$-folding decay time was $(2 \pi)^{-1} \mathrm{yr}$.

\section{Acknowledgements}

I would like to thank Klaus Hasselmann, Claude Frankignoul, Peter Lemke, and Tim Barnett for many helpful discussions. I am also grateful to Klaus Hasselmann for the statistical significance test which is presented in the appendix. I thank Jerome Namias and Robert Born for making available their NORPAX SST data set.

\section{Appendix}

The test for the validity of each model was determined by defining the probability distribution of $\varepsilon$ from (7) for a perfect fit with noise. Since the distribution could be assumed to be Gaussian, it is defined by the first and second moments. In this appendix, these moments are determined.

To begin, let $\bar{E}_{j}$ be the true spectrum and $\bar{a}_{k}$ the true fitted $k$ th parameter such that:

$\bar{E}_{j}=\hat{E}_{j}\left(\bar{a}_{k}\right)$,

where $E_{j} \equiv E\left(f_{j}\right)$ and where $k=1,2, \ldots, q$. The measured spectrum, $E_{j}$, differs from $E_{j}$ by the errors, $\delta E_{j}$ :

$E_{j}=\bar{E}_{j}+\delta E_{j}$, which results in fitted errors, $\delta a_{k}$ in $\bar{a}_{k}$ such that the actual fitted parameter, $a_{k}$, is:

$a_{k}=\bar{a}_{k}+\delta a_{k}$,

and the fitted spectrum is:

$\hat{E}_{j}\left(a_{k}\right)=\hat{E}_{j}\left(\hat{a}_{k}\right)+\delta E_{j}$,

where

$\delta E_{j}\left(a_{k}\right)=\sum_{k=1}^{q} \frac{\partial \hat{E}_{j}}{\partial a_{k}} \delta a_{k}$.

The next step is to express $\varepsilon$ in terms of these definitions by first rewriting (7) as:

$\varepsilon=\sum_{j=0}^{p-1}\left[\ln E_{j}-\ln \hat{E}_{j}\left(a_{k}\right)\right]^{2}$.

With the elimination of $E_{j}$ and $E_{j}$ by (A.2) and (A.3), and with the use of (A.1), (A.4) reduces to the first-order expression:

$\varepsilon=\sum_{j=0}^{p-1}\left(\frac{\delta E_{j}}{\hat{E}_{J}}-\frac{1}{\hat{E}_{J}} \sum_{k=1}^{q} \frac{\delta \hat{E}_{j}}{\partial a_{k}} \delta a_{k}\right)^{2}$.

To now find $\delta a_{k}$ in terms of $\delta E_{j}$, (A.4) is minimized to yield:

$0=\frac{\partial \varepsilon}{\partial a_{k}}=\sum_{j=0}^{\rho-1} 2\left(\ln E_{j}-\ln \hat{E}_{j}\right) \frac{1}{\hat{E}_{j}} \frac{\partial \hat{E}_{j}}{\partial a_{k}}$,

for each $k$. Equations (A.1)-(A.3) permit (A.6) to be reduced to the first-order expression:

$\mathbf{0}=\sum_{j=0}^{p-1}\left(\delta E_{j}-\sum_{l=1}^{q} \frac{\partial \hat{E}_{j}}{\partial a_{l}} \delta a_{l}\right) \frac{1}{\hat{E}_{j}^{2}} \frac{\partial \hat{E}_{j}}{\partial a_{k}}$.

With the definition of:

$m_{k l}=\sum_{j=0}^{p-1} \frac{1}{\hat{E}_{j}^{2}} \frac{\partial \hat{E}_{j}}{\partial a_{k}} \cdot \frac{\partial \hat{E}_{j}}{\partial a_{l}}$

as an element of the matrix, $M,(A .7)$ gives the desired result as:

$\delta a_{l}=\sum_{j=0}^{p-1} \sum_{k=1}^{q} m_{l k}^{-1} \frac{1}{\hat{E}_{j}^{2}} \frac{\partial E_{j}}{\partial a_{k}} \delta E_{j}$,

where $m_{l k}^{-1}$ is an element of the inverse of $M$.

The last intermediate step in finding the moments of the distribution of $\varepsilon$, is to determine the expectation values: $\left\langle\delta E_{j}\right\rangle$ and $\left\langle\delta E_{j}^{2}\right\rangle$. Since $v E_{j} / \bar{E}_{j}$ is distributed as a $\chi_{\nu}^{2}$-distribution (cf. Jenkins and

Tellus 30 (1978), 2 
Watts, 1968, p. 254) with $v$ degrees of freedom, and a mean and a variance of $v$ and $2 v$, respectively, then with the use of (A.2), the expectation values are:

$\left\langle\delta E_{j}\right\rangle=0$,

and

$\frac{\left\langle\delta E_{j}^{2}\right\rangle}{E_{j}^{2}}=\frac{2}{v}$.

To now determine the mean of the distribution of $\varepsilon$, it is necessary to eliminate $\delta a_{k}$ in (A.5) with the aid of (A.8) which yields:

$\varepsilon=\sum_{i=0}^{p-1} \sum_{j=0}^{p-1} R_{i j} \delta E_{i} \delta E_{j}$

where:

$R_{i j}=\frac{\delta_{i j}}{\hat{E}_{j}^{2}}-\frac{1}{\hat{E}_{i}^{2} \hat{E}_{j}^{2}} \sum_{k=1}^{q} \sum_{i=1}^{q} m_{l k}^{-1} \frac{\partial \hat{E}_{i}}{\partial a_{l}} \frac{\partial \hat{E}_{j}}{\partial a_{k}}$,

and $\delta_{i j}$ is the Kroneker delta. The expectation value of (A.11) can now be found to be:

$\langle\varepsilon\rangle=\sum_{j=0}^{p-1} R_{j j}\left\langle\delta E_{j}^{2}\right\rangle$,

since $\left\langle\delta E_{i} \delta E_{j}\right\rangle=0$ when $j \neq i$.

With the use of (A.10) and (A.12), this finally yields the desired mean:

$\langle\varepsilon\rangle=\frac{2}{v}(p-q)$.
To find the variance, (A.11) is first squared and then the expectation value is taken to give:

$$
\left\langle\varepsilon^{2}\right\rangle=\sum_{i=0}^{p-1} \sum_{j=0}^{p-1} \sum_{k=0}^{p-1} \sum_{l=0}^{p-1} R_{i j} R_{k l}\left\langle\delta E_{i} \delta E_{j} \delta E_{k} \delta E_{l}\right\rangle
$$

Since (A.15) was assumed to be Gaussian, then:

$$
\begin{aligned}
& \left\langle\delta E_{i} \delta E_{j} \delta E_{k} \delta E_{l}\right\rangle \cong\left\langle\delta E_{i} \delta E_{j}\right\rangle\left\langle\delta E_{k} \delta E_{l}\right\rangle \\
& \quad+\left\langle\delta E_{l} \delta E_{k}\right\rangle\left\langle E_{j} E_{l}\right\rangle+\left\langle\delta E_{i} \delta_{l}\right\rangle\left\langle\delta E_{j} \delta E_{k}\right\rangle
\end{aligned}
$$

(cf. Bendat and Piersol, 1966, p. 64) where $\left\langle\delta E_{j}\right\rangle=0$ by (A.9). Thus (A.15) reduces to:

$$
\left\langle\varepsilon^{2}\right\rangle=\sum_{i=0}^{p-1} \sum_{j=0}^{p-1}\left(R_{i i} R_{j j}+2 R_{i j}^{2}\right)\left\langle\delta E_{i}^{2}\right\rangle\left\langle\delta E_{j}^{2}\right\rangle .
$$

The variance can now be expressed as:

$$
\begin{aligned}
\left\langle(\varepsilon-\langle\varepsilon\rangle)^{2}\right\rangle & =\left\langle\varepsilon^{2}\right\rangle-\langle\varepsilon\rangle^{2} \\
& =2 \sum_{i=0}^{p-1} \sum_{j=0}^{p-1} R_{i j}^{2}\left\langle\delta E_{i}\right\rangle\left\langle\delta E_{j}\right\rangle,
\end{aligned}
$$

by (A.13) and (A.16). A final application of (A.10) and (A.12) permits (A.17) to be reduced to the desired expression for the variance:

$\left\langle(\varepsilon-\langle\varepsilon\rangle)^{2}\right\rangle=\frac{8}{v^{2}}(p-q)$.

The probability distribution of $\varepsilon$ is now defined by the mean (A.14) and the variance (A.18).

\section{REFERENCES}

Bendat, J. S. and Piersol, A. G. 1966. Measurement and analysis of random data. New York: John Wiley and Sons.

Copson, E. T. 1960. An introduction to the theory of functions of a complex variable. Oxford, Univ. Press.

Davis, R. E. 1976. Predictability of sea surface temperature and sea level pressure over the North Pacific Ocean. J. Phys. Ocean. 6, 249-266.

Frankignoul, C. and Hasselmann, K. 1977. Stochastic climate models, Part 2. Application to sea surface temperature anomalies and thermocline variability. Tellus 29, 289-305.

Hasselmann, K. 1976. Stochastic climate models, Part 1. Theory. Tellus 28, 473-485.

Jenkins, G. M. and Watts, D. G. 1968. Spectral analysis and its applications. San Francisco: Holden-Day.

Wunsch, C. 1972. Bermuda sea level in relation to tides, weather, and baroclinic fluctuations. Rev. Geophys. Space Phys. 10, 1-49. 


\section{АНОМАЛИИ ТЕМПЕРАТУРЫ ПОВЕРХНОСТИ В СЕВЕРНОЙ ЧАСТИ} ТИХОГО ОКЕАНА

Аномалии температуры поверхности в средних широтах северной части Тихого океана сравниваются с результатами предсказаний авторегрессионной модели первого порядка, в которой аномалии вызываются локальным белым шумом атмосферы. Результаты показывают, что модель может объяснить спектр мошности аномалий для чуть выше $50 \%$ исследуемых областей, главным образом, в центральных областях Тихого океана, но не работает, что неудивительно, в областях резких перемен или океанических фронтов. 